
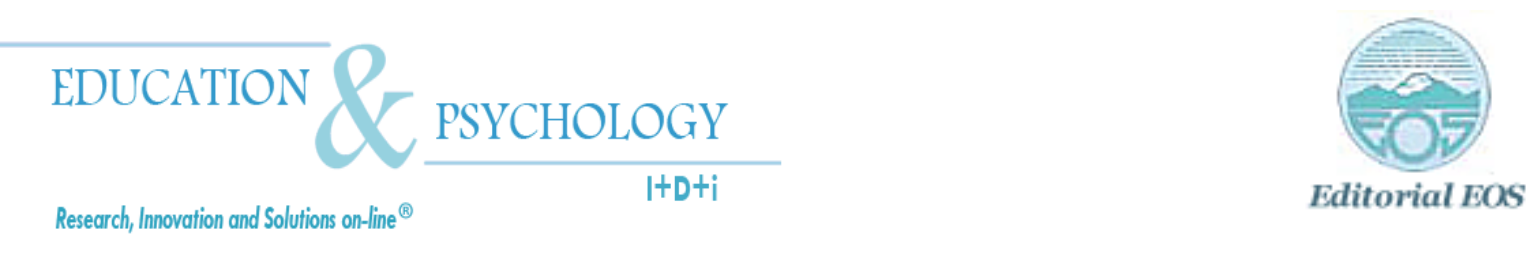

\title{
Preferencias respecto a métodos instruccionales de los estudiantes universitarios de nuevo acceso y su relación con estilos de aprendizaje y estrategias motivacionales
}

\section{Rafael García Ros, Francisco Pérez González, Isabel Talaya González}

Dpto. de Psicología Evolutiva y de la Educación, Universidad de Valencia

\section{España}

Rafael.Garcia@uv.es

Dr. Rafael García Ros. Universidad de Valencia, Dpto. Psicología Evolutiva y Educación, Facultad Psicología. Blasco Ibáñez, 21. 46010 Valencia. España. E-mail: rafael.garcia@uv.es

(C) Education \& Psychology I+D+i and Editorial EOS (Spain) 


\section{Resumen}

Introducción. El objetivo principal de este estudio es analizar las dimensiones que subyacen a las preferencias respecto a distintos métodos instruccionales de los estudiantes universitarios de nuevo acceso y su relación con sus estilos de aprendizaje y estrategias motivacionales.

Método. La muestra está compuesta por 158 estudiantes de primer año de Magisterio de la Universidad de Valencia (España). El estilo de aprendizaje fue evaluado a través del Inventory of Learning Processes, la orientación motivacional a través del Motivated Strategies for Learning Questionnaire y las preferencias respecto a métodos instruccionales a través de una escala específicamente diseñada para este trabajo.

Resultados. Los resultados destacan la existencia de tres tipos de preferencias: Métodos multidireccionales, unidireccionales y autónomos. Las preferencias por los métodos multidireccionales y unidireccionales resultan significativamente superiores a las preferencias por métodos de aprendizaje autónomo. Los resultados también destacan una asociación significativa, aunque de escasa magnitud, entre preferencias por métodos instruccionales, estilos de aprendizaje y orientación motivacional hacia el aprendizaje. Así, se observa una relación significativa entre preferencias por métodos multidireccionales y unidireccionales y el procesamiento elaborativo, así como una relación inversa entre preferencias por métodos multidireccionales y retención de hechos.

Conclusiones. Las orientaciones intrínseca y extrínseca hacia el aprendizaje están relacionadas con preferencias por métodos instruccionales distintos, las preferencias por métodos unidireccionales y autónomos se relacionan con una atribución interna de los resultados académicos, mientras que las preferencias por métodos multidireccionales se relacionan con altas expectativas de autoeficacia hacia el aprendizaje.

Palabras Clave: métodos de enseñanza, preferencias instruccionales, estilos de aprendizaje, orientación motivacional, instrucción adaptativa.

Recibido: 12/12/07 Aceptación Inicial: 31/03/08 Aceptación Definitiva: 03/05/08 


\begin{abstract}
Introduction. The main objective of this study is to analyze the dimensions underlying the preferences of university students regarding instructional methods, and the relationship of these preferences with their learning styles and motivational strategies.

Method. The sample consists of 158 students in their first year of teaching college at the University of Valencia (Spain). Learning style was evaluated using the Inventory of Learning Processes, motivational orientation by means of the Motivated Strategies for Learning Questionnaire, and preferences about instructional methods with a scale specifically designed for this study.

Results. The results highlight the existence of three types of preferences - multidirectional, unidirectional and autonomous - based fundamentally on the degree and type of interaction and level of autonomy each allows in academic learning. Preferences for multidirectional and unidirectional methods were significantly superior to preferences for autonomous learning. Furthermore, the results show a close relationship between preferences for multidirectional and unidirectional methods and elaborative processing, as well as an inverse relationship between the former and fact retention.
\end{abstract}

Conclusions. Preferences for unidirectional and autonomous methods are related to an internal attribution of the learning results, while preferences for multidirectional methods are related to high expectations of self-efficacy.

Keywords. teaching methods, instructional preferences, learning styles, motivational orientation, adaptive instruction.

Received: 12/12/07_Initial Acceptance: 31/03/08_ Final Acceptance: 03/05/08 


\section{Introducción}

La metodología instruccional ha constituido tradicionalmente un foco de especial interés para los investigadores educativos. Así, los modelos sobre situaciones educativas, entendidos como propuestas descriptivas y de organización funcional de las variables que intervienen en los procesos de enseñanza/aprendizaje, incluyen los métodos de enseñanza como un elemento fundamental en los mismos (Anderson y Burns, 1989; Entwistle, 1988; Fraser, 1987; Rivas, 1997). Las teorías instruccionales, eminentemente prescriptivas y orientadas hacia la práctica educativa, identifican métodos útiles para favorecer los procesos de aprendizaje y las situaciones en que dichos métodos resultan pertinentes (Reigeluth, 1999). Por último, los modelos de diseño instruccional -que asumen una perspectiva eminentemente tecnológica y son entendidos como procedimientos sistemáticos para guiar la planificación, implementación y control-evaluación de los procesos de enseñanza/aprendizaje (Gufstafson y Branch, 2002)- también incluyen como elemento crucial la decisión sobre qué estrategias o métodos instruccionales seleccionaremos para facilitar los procesos de aprendizaje (p.e., Newby, Stepich, Lehman y Russell, 2000; Podolskij, 1997; Terlouw, 1997).

La investigación sobre diferencias individuales también goza de una amplia tradición en el campo instruccional, y ha centrado gran parte de sus esfuerzos en analizar las relaciones entre características individuales de los actores -aprendices y profesores- de los procesos educativos (p.e., conocimientos previos, estilos y estrategias de aprendizaje, características de personalidad, ...) y de las variables del contexto de enseñanza (p.e., características y complejidad de los contenidos, tiempo y recursos disponibles, tipos de agrupamiento). En este marco surgió el concepto de "instrucción adaptativa" (Corno y Snow, 1986), entendida como la necesidad de responder a las diferencias individuales de los actores de los procesos educativos, facilitando al profesor adecuar su actuación a las características de los alumnos, o ayudando a los alumnos a ajustar sus expectativas y comportamientos a las particularidades de los docentes. Los estudios dirigidos a evaluar la interacción entre métodos instruccionales y variables del contexto instruccional (especialmente tipo de contenidos) resultan muy numerosos y, sin embargo, resulta muy inferior la investigación que analiza las relaciones entre características de los aprendices y/o profesores con las preferencias por distintas metodologías (Clariana, 2001).

Es en esta última línea donde centramos nuestro trabajo, dirigido a analizar las preferencias de los estudiantes de magisterio de nuevo acceso hacia los distintos métodos instruc- 
cionales. Por preferencias instruccionales consideramos "la tendencia de los individuos a elegir o expresar preferencias por una técnica o combinación de técnicas de enseñanza determinadas" (Sadler-Smith, 1996, p. 31) y, por métodos instruccionales "las distintas vías o procedimientos para ayudar a los estudiantes a conseguir los objetivos de aprendizaje o adquirir los contenidos de enseñanza" (Newby, Stepich, Lehman y Russell, 2000, p. 91). De este modo, nuestro objetivo no es determinar si un método es mejor que otro -cuestión que dependerá de la interacción entre las variables anteriormente destacadas (profesor, aprendices y contexto)-, sino conocer las preferencias de los aprendices hacia uno o varios métodos instruccionales.

Son muchas las clasificaciones sobre métodos instruccionales que podemos encontrar en la literatura científica en función de diversos tipos de criterios (Reigeluth y Moore, 1999): dominio (cognitivo, psicomotor, actitudinal), tipo de aprendizaje (recuerdo, comprensión y/o aplicación), control del aprendizaje (centrados en el profesor o en el alumno), dirección del aprendizaje (de ámbito específico frente a multidisciplinares, orientados a contenidos o a problemas), tipo de agrupamiento, interacciones para aprender y/o apoyos para el aprendizaje (cognitivo y emocional). También resulta tradicional la diferencia entre métodos expositivos que enfatizan la presentación ordenada y exhaustiva de los contenidos de aprendizaje- y por descubrimiento -que inciden en la exploración, elaboración y comprobación personal de la información - (Romiszowski, 1981). Otros autores (p.e., Rivas 1997) ofrecen clasificaciones en función del tipo de conocimiento a adquirir -declarativo (clase magistral, explicación, demostración e interrogación), procedimental (tutoría y puesta en común) y condicional (debate, estudio de casos, talleres, laboratorio, seminario)- y/o en función la direccionalidad de la comunicación -unidireccionales (clase magistral, exposición, demostración, interrogación y tutoría), multidireccionales (centrados en la dirección y control de la dinámica de clase -puesta en común, debate, mesa redonda y panel- y centrados en la interacción y control del trabajo taller, estudio de casos, trabajo de laboratorio, trabajo de campo y seminario de investigación). Por último, Hernández (1998) clasifica los distintos métodos en función de las siguientes dimensiones: (a) perspectiva logocéntrica-psicocéntrica (en función de si toman como eje central de la práctica docente los objetivos-contenidos de enseñanza o los conocimientos previos, experiencias personales, esquemas y motivaciones de los aprendices), (b) verbales frente a naturales/simulación, (c) roles del profesor-aprendiz (métodos expositivos-receptivos, métodos interactivos y métodos activos de entrenamiento-práctica y descubrimiento). 
Pese a que las preferencias instruccionales de los estudiantes constituye un tema tradicional en el campo de la investigación psicoeducativa (Ausburn y Brown, 2006; Check, 1984; Entwistle y Peterson, 2005; Ford y Chen, 2001; James, 1962; Lowyck, Elen y Clarebout, 2005; Phillips, 1999; Tait, Entwistle y McCune, 1998), y que en los últimos años resultan muy numerosos los trabajos centrados en las preferencias de los aprendices respecto a la utilización de nuevas tecnologías en los procesos de enseñanza/aprendizaje (p.e., Shuell y Farber, 2001; Smith, 2001; van den Bosch, 2006), resultan mucho menos numerosos los que se centran específicamente en analizar las preferencias de los aprendices universitarios respecto a los métodos instruccionales, así como aquellos que pretenden evaluar su relación con sus estilos de aprendizaje y orientación motivacional.

Entre estos últimos trabajos se puede destacar el realizado por Hocevar, Zimmer y Strom (1987) que obtienen dos dimensiones relativas a preferencias en función del (1) grado de estructuración y (2) niveles de dificultad de las actividades propuestas. En un contexto más próximo, y con estudiantes de Enseñanza Secundaria, Clariana (2001) analiza la relación entre preferencias por métodos basados en el aprendizaje por recepción frente al aprendizaje por descubrimiento y distintas variables sociopersonales (edad, sexo, cognitivas y de personalidad) y escolares (rendimiento), constatando la ausencia de diferencias significativas en cuanto a preferencias hacia uno u otro tipo de métodos, la relación entre nivel de rendimiento en materias de letras y preferencias por métodos receptivos, así como entre extraversión y preferencias por métodos por descubrimiento.

Entre los trabajos que centran su atención en analizar la relación entre estilos de aprendizaje y preferencias por métodos instruccionales de los universitarios destacan los elaborados por Sadler-Smith (1997) y Sadler-Smith y Riding (1999). En el primero, Sadler (1997) analiza las preferencias de 245 estudiantes de ciencias empresariales por nueve métodos instruccionales diferentes a través del Inventory of Learning Preferences, así como su relación con los estilos de aprendizaje evaluados a través del Learning Styles Questionnaire (Honey y Mumford, 1986; 1992) -estilos activo, reflexivo, teórico y pragmático-. En referencia a las preferencias por los métodos instruccionales sus resultados sugieren la existencia de tres grandes factores subyacentes: (a) métodos autónomos (aprendizaje asistido por ordenador, aprendizaje a distancia/flexible), (b) métodos colaborativos (role-playing, grupos de discusión y juegos instruccionales) y, (c) métodos dependientes (lección magistral, tutoría/consulta). Sin especificar en su trabajo la significatividad o no de las diferencias encontra- 
das, la valoración de los métodos dependientes (media $=3.92$, d.s. $=.9$ ) es superior a la de los métodos colaborativos (media $=3.22$, d.s. $=.9$ ) y a la de los métodos autónomos (media $=3.07$, d.s.=1.0). En cuanto a la relación entre preferencias y estilos de aprendizaje, obtiene relaciones significativas aunque de escasa magnitud entre las subescalas de ambos instrumentos, obteniendo niveles de asociación relevantes exclusivamente entre preferencias por métodos colaborativos y estilo activo $(\mathrm{r}=.24, \mathrm{p}<.001)$, preferencias por métodos colaborativos y estilo reflexivo $(\mathrm{r}=-.15, \mathrm{p}<.05)$, preferencias por métodos autónomos y estilo reflexivo $(\mathrm{r}=.17$, $\mathrm{p}<.05$ ). Estos resultados apoyan la inexistencia de una relación importante entre estilos de aprendizaje y preferencias instruccionales evaluadas a través de los dos instrumentos destacados.

En el segundo estudio con 240 estudiantes de ciencias empresariales, Sadler-Smith y Riding (1999) amplian el alcance del Inventory of Learning Preferences incorporando ítems relativos a preferencias por medios instruccionales y técnicas de evaluación. Respecto a las preferencias por métodos instruccionales destacan de nuevo la existencia de tres dimensiones subyacentes a las mismas (métodos colaborativos, dependientes y autónomos) y, adicionalmente, identifican la relación entre estilo cognitivo analítico-global y las preferencias por métodos colaborativos, siendo los sujetos caracterizados por un estilo global (media=3.34, d.s. $=0.9)$ frente a los analíticos $($ media $=3.09$, d.s. $=0.9)$ quienes muestran mayor preferencia por este tipo de métodos.

Garner y Korth (1998) analizan la relación entre los estilos de aprendizaje derivados del modelo de aprendizaje experimental de $\operatorname{Kolb}(1984,1985)$ y las actitudes hacia diversos métodos de aprendizaje -clases tradicionales, elaboración de informes, efectuar lecturas, aprendizaje por pares y trabajo en grupo-, constatando la relación entre ambos constructos. De forma más concreta, los sujetos asimiladores - caracterizados por la conceptualización abstracta, por el análisis lógico de la información y por una presentación concisa de la misma- prefieren las clases tradicionales y la elaboración de informes, mientras que los acomodadores caracterizados por aprender desde las experiencias concretas y la experimentación activa, así como desde la intución frente al análisis lógico- preferían los trabajos en grupo. Más recientemente, Loo (2004) analiza la relación entre los cuatro estilos de aprendizaje definidos por el modelo de Kolb - divergentes, convergentes, asimiladores y acomodadores- y las preferencias de un grupo de estudiantes universitarios respecto a 12 elementos instruccionales. Los resultados destacan una relación significativa, aunque también de escasa magnitud, entre estilos de 
aprendizaje y preferencias instruccionales. Así, independientemente de su estilo de aprendizaje, todos los sujetos manifestaban preferencias por efectuar ejercicios prácticos, resolver problemas y participar en grupos, mientras que mostraban valoraciones muy inferiores a la redación de informes, efectuar presentaciones a sus compañeros y/o revisar documentos.

En la línea destacada en párrafos precedentes, los objetivos fundamentales de nuestro trabajo son (a) evaluar las preferencias respecto a métodos instruccionales de estudiantes de nuevo acceso de la titulación de Magisterio, (b) analizar las dimensiones que subyacen a las valoraciones de las preferencias de los aprendices y, (c) analizar las relaciones existentes entre preferencias instruccionales, estilos de aprendizaje y estrategias motivacionales.

\section{Método}

\section{Participantes}

En el estudio han participado 158 estudiantes de nuevo acceso de la titulación de Magisterio de la Universidad de Valencia. La edad de este grupo de estudiantes oscila entre los 18 y los 40 años, siendo la media de 19.5 años con una desviación típica de 0.42 . El 79\% de los sujetos son mujeres y el $21 \%$ hombres, y presentan un nivel socioeconómico medio y medio-bajo. El grupo de estudiantes presenta diversas áreas de especialización (educación infantil, primaria, música, educación física, lengua extranjera, y audición y lenguaje).

\section{Instrumentos y procedimiento}

\section{Escala de Preferencias respecto a Métodos Instruccionales}

La Escala de Preferencias respecto a Métodos Instruccionales utilizada en este estudio parte de la revisión de estudios precedentes sobre la temática (Marqués, 2000; Sadler-Smith y Riding, 1999) y de manuales sobre teorías (p.e., Reigeluth, 1999) y tecnología instruccional (Newby et al., 2000). Tras obtener una versión preliminar de la misma, ésta fue sometida a un grupo de discusión integrado por los componentes del grupo de investigación, psicólogos escolares y profesores de enseñanza secundaria, con el objetivo de determinar los ítems específicos a incorporar en la escala. Tras la discusión sobre la relevancia de los ítems planteados, se llegó al acuerdo unánime de plantear una versión de la escala compuesta por 13 ítems (véase en tabla 1). Ésta fue la que sometimos a evaluación utilizando una escala con diez niveles de respuesta -desde "En absoluto preferido" (1) hasta "Totalmente preferido" (10)-, por ser la comúnmente utilizada en el sistema educativo español. Adicionalmente, y con el objetivo de 
evitar ambigüedades y/o creencias erróneas se proporcionó una descripción de cada uno de los métodos instruccionales que se integran en la escala.

\section{Inventory of Learning Processes (ILP)}

Como instrumento de evaluación de los estilos de aprendizaje se utiliza una adaptación española del Inventory of Learning Processes (Schmeck, 1983; Schmeck, Ribich y Ramanaiah, 1977, 1978), cuya bondad psicométrica y validez factorial ha sido constatada en distintos estudios con población española (Cano y Justicia, 1993; García-Ros, PérezGonzález, Martínez y Alfonso, 1999). Schmeck conceptualiza los estilos de aprendizaje como "una predisposición a adoptar una estrategia de aprendizaje particular independientemente de las demandas de las tareas de aprendizaje" (Schmeck, 1983, p. 233), que es "considerada como una extensión entre la personalidad y las estrategias de aprendizaje que utiliza el sujeto sobre un continuo causal que conduce a un resultado de aprendizaje” (Schmeck, 1988, p. 174). La prueba aplicada consta de 36 ítems que, utilizando una escala tipo Likert con cinco niveles de respuesta (desde 1 -nunca- a 5 -siempre-), evalúa las cuatro dimensiones complementarias destacadas a continuación:

- Procesamiento Profundo (PROF). Integra 9 items y se asocia con estrategias de conceptualización y búsqueda de significados, de comparación y contraste de abstracciones, así como de evaluación crítica de la información.

- Procesamiento Elaborativo (ELA). Integra 12 items que evalúan las preferencias por la elaboración y personalización de la información y del material de clase.

- Retención de Hechos (RET_H). Incorpora 4 items y se relaciona con la preferencia por la información de hechos y el recuerdo de detalles.

- Estudio Metódico (MET). Integra 11 items y evalúa el uso sistemático de las técnicas de estudio tradicionales.

\section{Motivated Strategies for Learning Questionnaire (MSLQ)}

El MSLQ constituye una de las pruebas de mayor prestigio para evaluar el aprendizaje autorregulado y su objetivo es "evaluar la orientación motivacional de estudiantes universitarios y el uso de diferentes estrategias de aprendizaje en un curso" (Pintrich, Smith, Garcia y McKeachie, 1991, p. 3). Numerosas y recientes investigaciones en nuestro país destacan la importancia del aprendizaje autorregulado como competencia básica a desarrollar en el sistema educativo, centrándose tanto en la dimensión estratégica como motivacional (p.e., De la 
Fuente y Justicia, 2007; Monereo, 2007). En este estudio utilizamos la categoría motivacional de la adaptación española del MSLQ (Reynaldo y Galán, 2000; Roces, Tourón, González, Núñez, González y García, 1993), que evalúa las dimensiones de orientación a metas intrínsecas (OI), orientación a metas extrínsecas (OE) y valor de la tarea (VT), creencias de control del aprendizaje (CREEN), autoeficacia para el aprendizaje y la ejecución (AUTOEF), y ansiedad ante los exámenes (ANS)-. La prueba aplicada consta de 31 items con una escala tipo Likert con siete niveles de respuesta (desde 1 -en absoluto- a 7 -completamente). Los instrumentos fueron cumplimentados durante el horario académico. Todas las dudas fueron consideradas y atendidas individualmente.

\section{Análisis}

En primer lugar, se realizó un Análisis Factorial Exploratorio con rotación Varimax utilizando el programa SPSS 10.0.6 para Windows, con el objetivo de evaluar la dimensionalidad de las respuestas proporcionadas en la Escala de Preferencias respecto a Métodos Instruccionales. En segundo lugar, evaluamos los estadísticos descriptivos y consistencia interna (coeficiente alpha) de las subescalas de preferencias instruccionales surgidas del análisis precedente. Por último, llevamos a cabo un análisis correlacional (correlación de Pearson bilateral), con el objetivo de analizar la relación entre las preferencias por métodos instruccionales, los estilos de aprendizaje y la orientación motivacional de los estudiantes.

\section{Resultados}

Escala de Preferencias respecto a Métodos Instruccionales

\section{Análisis de items}

En la tabla 1 presentamos los descriptivos básicos para cada uno de los ítems que componen la escala, así como la comunalidad entre los mismos. Los descriptivos básicos de los ítems muestran que los métodos más valorados son "Práctica y ejercitación”, "Aprendizaje Cooperativo", "Demostraciones", "Simulaciones" y "Juegos Instruccionales". Por otro lado, "Aprendizaje a Distancia" presenta el valor inferior (2.56), muy por debajo de la media teórica de referencia (5.0); “Aprendizaje a través de Internet”, "Exposición o lección magistral" y "Aprendizaje asistido por ordenador" presentan valores ligeramente por debajo de la media teórica (medias entre 4.4 y 4.7). En cuanto a las comunalidades, todas alcanzan valores superiores al valor crítico tradicionalmente considerado $(0.30)$. 
Tabla 1. Descriptivos básicos e índices de comunalidad de los ítems de la Escala de Preferencias respecto a Métodos Instruccionales

\begin{tabular}{|l|c|c|c|}
\hline Ítems & Media & d.t. & Comunalidad \\
\hline 1.- Exposición o lección magistral & 4.70 & 2.59 & .48 \\
\hline 2.- Grupos de discusión & 6.98 & 2.09 & .45 \\
\hline 3.- Aprendizaje asistido por ordenador & 4.70 & 2.50 & .77 \\
\hline 4.- Simulaciones & 7.59 & 2.18 & .44 \\
\hline 5.- Role-playing & 6.40 & 2.57 & .47 \\
\hline 6.- Aprendizaje a distancia & 2.56 & 1.82 & .48 \\
\hline 7.- Tutorización individual cara a cara & 5.59 & 2.66 & .32 \\
\hline 8.- Aprendizaje cooperativo & 7.91 & 1.81 & .56 \\
\hline 9.- Juegos instruccionales & 7.20 & 2.19 & .46 \\
\hline 10.- Resolución de problemas & 6.61 & 2.16 & .57 \\
\hline 11.- Demostraciones & 7.64 & 1.89 & .58 \\
\hline 12.- Práctica y ejercitación & 8.09 & 2.06 & .33 \\
\hline 13.- Aprendizaje a través de Internet & 4.49 & 2.82 & .77 \\
\hline
\end{tabular}

\section{Análisis Factorial Exploratorio}

Los resultados iniciales que ofrece el análisis factorial de componentes principales destacan la adecuación de los datos para la aplicación de este tipo de prueba estadística, dado que la medida de adecuación muestral de Kaiser-Meyer-Olkin presentó un valor de 0.67, así como la prueba de esfericidad de Bartlett $\left(\chi^{2}(78)=518.05, \mathrm{p}<.0001\right)$. El método de Kaiser sugirió la existencia de tres factores subyacentes que explican conjuntamente el $50.62 \%$ de la varianza de las puntuaciones. La tabla 2 presenta las saturaciones de cada uno de los items en los factores correspondientes, así como los valores propios y el porcentaje de varianza explicada por los mismos.

Utilizando como punto de corte para incorporar un ítem a un factor una saturación de 0.40, la solución factorial queda definida del siguiente modo:

- Métodos multidireccionales (MULT-DIR). Integrado por los items 1 (con saturación negativa), 2, 4, 5, 8 y 9. Su valor propio es 2.38 y explica un $17.9 \%$ de la varianza de las respuestas al cuestionario. El valor promedio de los items de la subescala es 7.07 . 
- Métodos unidireccionales (UNI-DIR). Integrado por los items 7, 10, 11 y 12. Su valor propio es 2.13 y explica el $16.4 \%$ de la varianza de los datos. El valor promedio de los items de la subescala es 6.97.

- Métodos autónomos (AUTON). Integrado por los items 3, 6 y 13. Su valor propio es 2.12 y explica el $16.3 \%$ de la varianza de los datos. El valor promedio de los items de la subescala es 3.94 .

Tabla 2. Saturaciones factoriales de los ítems de la Escala de Preferencias respecto a Métodos Instruccionales

\begin{tabular}{|l|c|c|c|}
\hline \multirow{2}{*}{ Items } & \multicolumn{3}{|c|}{ Factores } \\
\cline { 2 - 4 } & $\mathbf{1}$ & $\mathbf{2}$ & $\mathbf{3}$ \\
\hline 1.- Exposición o lección magistral & -.614 & .322 & .019 \\
\hline 2.- Grupos de discusión & .660 & .003 & .009 \\
\hline 3.- Aprendizaje asistido por ordenador & .128 & -.119 & .862 \\
\hline 4.- Simulaciones & .569 & .109 & .325 \\
\hline 5.- Role-playing & .585 & .335 & .113 \\
\hline 6.- Aprendizaje a distancia & -.231 & .007 & .653 \\
\hline 7.- Tutorización individual cara a cara & .022 & .438 & -.152 \\
\hline 8.- Aprendizaje cooperativo & .681 & .306 & .069 \\
\hline 9.- Juegos instruccionales & .474 & .322 & .230 \\
\hline 10.- Resolución de problemas & .066 & .744 & .112 \\
\hline 11.- Demostraciones & .086 & .759 & .023 \\
\hline 12.- Práctica y ejercitación & .188 & .537 & .042 \\
\hline 13.- Aprendizaje a través de Internet & .205 & .050 & .854 \\
\hline Valor Propio & 2.38 & 2.13 & 2.12 \\
\hline \% Varianza explicada & 17.9 & 16.4 & 16.3 \\
\hline
\end{tabular}

\section{Correlación entre factores y consistencia interna}

La tabla 3 presenta las correlaciones de Pearson entre las puntuaciones de los distintos factores de la Escala de Preferencias respecto a Métodos Instruccionales, así como los niveles de consistencia interna de cada una de las subescalas que integra.

Como podemos observar, existe una correlación positiva significativa entre las preferencias por métodos unidireccionales y multidireccionales $(\mathrm{r}=.29, \mathrm{p}<.001)$, mientras que lascorrelaciones de ambas con el factor de preferencias por métodos autónomos son prácticamente nulas. La consistencia interna (alfa de Cronbach) de los factores de preferencias por métodos multidireccionales y métodos unidireccionales es de 0.69 en ambos casos, mientras que la del factor de preferencias por métodos autónomos es de 0.55 . 
Tabla 3. Nivel de asociación entre factores de preferencias respecto a métodos instruccionales

\begin{tabular}{|l|c|c|c|}
\hline \multicolumn{1}{|c|}{ Factores } & Multidireccionales & Unidireccionales & Autónomos \\
\hline Multidireccionales & .69 & & \\
\hline Unidireccionales & $.29^{* * *}$ & .69 & \\
\hline Autónomos & .07 & .03 & .55 \\
\hline
\end{tabular}

Nivel significación (bilateral): ${ }^{* * *} .001, * * .01, * .05$. Coeficientes Alpha en la diagonal.

\section{ANOVA de medidas repetidas}

Con el objetivo de constatar la superioridad de las preferencias por unos u otros métodos instruccionales se efectuó un ANOVA de medidas repetidas, comparando las valoraciones que cada uno de los sujetos destacaba respecto a los métodos multidireccionales, unidireccionales y autónomos. Una vez constatado el incumplimiento del supuesto de esfericidad a través de la prueba de Mauchly $\left(\mathrm{W}_{\text {Mauchly }}=.85\right.$, ji-cuadrado $\left.(2)=25.2, \mathrm{p}<.001\right)$ y considerando la utilización del corrector epsilon de Greenhouse-Geisser $\left(\varepsilon_{\mathrm{GG}}=.87\right)$, los resultados destacan la existencia de diferencias significativas entre las preferencias expresadas por los sujetos respecto a los distintos métodos instruccionales considerados $-\mathrm{F}_{1.74,273.3}=241, \mathrm{p}<.001-$. De forma más específica, las comparaciones a posteriori a través de la prueba de Bonferroni señalan que resultan significativamente superiores las preferencias por los métodos multidireccionales y unidireccionales frente a los autónomos (diferencia de medias multidireccionalesautónomos $=3.14, \mathrm{p}<.001$; diferencia de medias unidireccionales-autónomos=3.03, $\mathrm{p}<.001$ ), mientras que los dos primeros presentan niveles homogéneos entre sí (diferencia de medias= $0.1, \mathrm{p}<.98)$.

\section{Preferencias respecto a métodos instruccionales y estilos de aprendizaje}

La tabla 4 presenta las correlaciones de Pearson, y su correspondiente significación, entre las puntuaciones de los factores de la escala desarrollada para este estudio y la adaptación española del Inventario de Procesos de Aprendizaje (ILP). 
Tabla 4. Correlaciones entre Preferencias respecto a Métodos Instruccionales y

Estilos de Aprendizaje

\begin{tabular}{|l|c|c|c|c|}
\cline { 2 - 5 } \multicolumn{1}{c|}{} & \multicolumn{4}{c|}{ Inventario de Procesos de Aprendizaje (ILP) } \\
\cline { 2 - 5 } \multicolumn{1}{c|}{} & PROF & MET & RET_H & ELA \\
\hline MET. MULT-DIR & .01 & -.06 & $-.22^{* *}$ & $.21^{* *}$ \\
\hline MET. UNI-DIR & .04 & .02 & -.00 & $.24^{* *}$ \\
\hline MET. AUTON & -.02 & -.02 & -.07 & .02 \\
\hline
\end{tabular}

Nivel de significación (bilateral): ***.001, **.01, *.05.

El factor de preferencias por métodos multidireccionales presenta correlaciones significativas con retención de hechos en sentido inverso $(\mathrm{r}=-.22, \mathrm{p}<.01)$ y con procesamiento elaborativo en sentido directo $(\mathrm{r}=.21, \mathrm{p}<.01)$. El factor de preferencias por métodos unidireccionales correlaciona positivamente con procesamiento elaborativo $(\mathrm{r}=.24, \mathrm{p}<.01)$. Por último, el factor de preferencias por métodos autónomos no correlaciona significativamente con ninguna dimensión relativa a estilos de aprendizaje.

\section{Preferencias respecto a métodos instruccionales y orientación motivacional}

La tabla 5 presenta las correlaciones de Pearson, y su correspondiente significación, entre las puntuaciones de los factores obtenidos en la Escala de Preferencias respecto a Métodos Instruccionales y los correspondientes a Orientación Motivacional evaluados a través de la adaptación española del MSLQ.

El factor de preferencias por métodos multidireccionales presenta correlaciones significativas con las dimensiones de orientación intrínseca $(r=.23, p<.01)$ y autoeficacia para el aprendizaje y la ejecución $(\mathrm{r}=.17, \mathrm{p}<.05)$. El factor de preferencias por métodos unidireccionales correlaciona significativamente con orientación intrínseca $(\mathrm{r}=.22, \mathrm{p}<.01)$, orientación extrínseca $(\mathrm{r}=.15, \mathrm{p}<.05)$ y con creencias sobre el control del aprendizaje $(\mathrm{r}=.16, \mathrm{p}<.05)$. El factor de preferencias por métodos autónomos correlaciona únicamente con creencias sobre el control del aprendizaje $(\mathrm{r}=.17, \mathrm{p}<.05)$. No se obtiene nivel de asociación significativo alguno entre preferencias por métodos instruccionales y las dimensiones motivacionales relativas a ansiedad ante las evaluaciones y valor de la tarea. 
Tabla 5. Correlaciones entre Preferencias respecto a Métodos Instruccionales y Orientación Motivacional hacia el Aprendizaje

\begin{tabular}{|l|c|c|c|c|c|c|}
\cline { 2 - 7 } \multicolumn{1}{c|}{} & \multicolumn{6}{c|}{ Orientación Motivacional hacia el Aprendizaje (MSLQ) } \\
\cline { 2 - 7 } \multicolumn{1}{c|}{} & OI & OE & VT & CREEN & AUTOEF & ANS \\
\hline Multidireccionales & $.23^{* *}$ & -.04 & .11 & .13 & $.17^{*}$ & -.01 \\
\hline Unidireccionales & $.22^{* *}$ & $.15^{*}$ & .08 & $.16^{*}$ & .00 & -.002 \\
\hline Autónomos & -.05 & .02 & -.02 & $.17^{*}$ & -.01 & .08 \\
\hline
\end{tabular}

Nivel de significación (bilateral): ***.001, **.01, *.05.

\section{Conclusiones y discusión}

Los resultados indican que las preferencias de los estudiantes de nuevo acceso de la titulación de magisterio se decantan hacia métodos como "Práctica y ejercitación", "Aprendizaje Cooperativo", "Demostraciones", "Simulaciones" y "Juegos Instruccionales". Por el contrario, el método peor valorado es "Aprendizaje a distancia" con un valor muy por debajo de la media teórica. Adicionalmente, el análisis factorial de las preferencias de los estudiantes hacia los distintos métodos instruccionales considerados destaca la existencia de tres grandes dimensiones subyacentes:

a) Métodos multidireccionales. Caracterizados por estar centrados en el aprendiz y requerir un alto grado de interacción entre los aprendices en el desarrollo de las actividades académicas. Incluye los métodos de "exposición o lección magistral" (saturación negativa), "grupos de discusión", "simulaciones", "role-playing", “aprendizaje cooperativo" y "juegos instruccionales".

b) Métodos unidireccionales. Constituido por los métodos instruccionales más tradicionales centrados en el profesor, caracterizados por la interacción y transmisión de la información en el sentido profesor-alumno. Incluye los métodos de "tutorización individual", "resolución de problemas", "demostraciones" y "práctica y ejercitación".

c) Métodos autónomos. Caracterizados por un alto grado de autonomía y un menor nivel de interacción social. Incluye los métodos de "aprendizaje asistido por ordenador", "aprendizaje a distancia" y "aprendizaje a través de Internet”. 
En función de estos resultados se puede destacar que la estructura subyacente a las preferencias sobre metodología instruccional se articula fundamentalmente en torno a tres de las dimensiones destacadas por Reigeluth y Moore (1999): ubicación del control del aprendizaje (profesor versus aprendiz), grado y tipo de interacción implicados y tipo de agrupamiento típico que facilita. Así, los métodos multidireccionales y unidireccionales se caracterizarían por su elevado nivel de interactividad, aunque con diferencias en el eje de la interacción (alumno-alumno o profesor-alumno), y serían adecuados para pequeños-medianos grupos de trabajo, mientras que los métodos autónomos se caracterizarían fundamentalmente por un elevado nivel de autonomía del aprendiz y por una actividad de tipo individual.

Por otro lado, de forma similiar a los resultados obtenidos en trabajos previos (SadlerSmith, 1997) se ha constatado la superioridad de las preferencias por los métodos multidireccionales y los unidireccionales - con niveles muy similares entre sí en nuestro caso- frente a los métodos autónomos. Estos resultados son congruentes con las características de los estudiantes participantes en el estudio, dado que han accedido a una universidad de carácter presencial frente a otras alternativas basadas en el aprendizaje a distancia y, adicionalmente, al tratarse de estudiantes de nuevo acceso su nivel de experiencia previa con las nuevas tecnologías en educación se limita a la obtenida en la Educación Secundaria. En este sentido, se considera de interés analizar en trabajos futuros si las preferencias por los métodos instruccionales varían en función de la elección de la modalidad de estudios universitarios (presencial frente a distancia) y, más específicamente, evaluar la relación entre las preferencias por los métodos autónomos y el uso/dominio de las nuevas tecnologías por parte de los aprendices, así como con la experiencia y familiarización con las mismas en las etapas previas del sistema educativo.

Pese a utilizar escalas sensiblemente distintas, los resultados destacados hasta el momento coinciden básicamente con las señaladas por Sadler-Smith (1997) y Sadler-Smith y Riding (1999), tanto respecto a las dimensiones subyacentes a las preferencias respecto a métodos instruccionales (multidireccionales, unidireccionales y autónomos), como a las características definitorias de las mismas (nivel de interacción y grado de autonomía) y a la superioridad de las preferencias por los métodos multidireccionales y unidireccionales frente a los autónomos. Las principales discrepancias se centran en que las preferencias por los métodos autónomos resulta sensiblemente inferiores en nuestro caso (en el estudio de Sadler-Smith y Riding supera la media teórica de la escala de respuesta) y en que el método “exposición o 
lección magistral" queda ubicado en nuestro estudio con saturación negativa en la escala de preferencias por métodos multidireccionales, mientras que en los dos trabajos citados se sitúa en métodos unidireccionales.

Aunque cabe analizar con mayor detalle estas dos discrepancias, las diferencias en la valoración de los métodos autónomos podría estar provocada por factores diferenciales de tipo contextual -experiencia y familiarización con el uso de nuevas tecnologías en la enseñanza obligatoria en ambos paises- y/o relacionadas con las características de los sujetos participantes en ambos estudios -en nuestro caso se trata de estudiantes de nuevo acceso en la titulación de magisterio, mientras que en los trabajos destacados participan sujetos de cursos más avanzados en titulaciones de ciencias empresariales-. Por otro lado, pese a que nuestros resultados sugieren la ubicación del método "exposición o lección magistral” en el factor preferencias por métodos multidireccionales -dada la magnitud de su saturación negativa en el factor mencionado- frente al factor preferencias por métodos unidireccionales, esta ubicación deberá ser confirmada en estudios posteriores, tanto desde un punto de vista conceptual como empírico - este ítem también satura en el factor métodos unidireccionales con un valor superior al tradicionalmente considerado de .30-

En cuanto a la relación entre preferencias por métodos instruccionales y estilos de aprendizaje, hemos constatado la existencia de relaciones significativas de escasa magnitud exclusivamente entre preferencias por métodos multidireccionales y procesamiento elaborativo $(\mathrm{r}=.21, \mathrm{p}<.01)$, entre métodos unidireccionales y procesamiento elaborativo $(\mathrm{r}=.24, \mathrm{p}<.01)$ y, por último, entre preferencias por métodos multidireccionales y retención de hechos $(\mathrm{r}=-$ $.22, \mathrm{p}<.01)$. No se ha obtenido relación significativa alguna entre preferencias y las dimensiones de estilos de aprendizaje relativas a procesamiento profundo y estudio metódico. Estos resultados coindicen básicamente con los destacados en trabajos precedentes que, aunque partiendo de modelos teóricos distintos sobre los estilos de aprendizaje, señalan la inexistencia de una relación importante entre ambos constructos (Loo, 2004; Sadler-Smith, 1997).

De forma más específica, la dimensión de estilos de aprendizaje Procesamiento Profundo -así denominada por su relación con los niveles de profundidad del procesamiento establecidos por Craik y Lockhart (1972) que implica una aproximación al aprendizaje basada en la conceptualización y en la búsqueda de significados, en la comparación y contraste de fuentes de información, en la categorización y evaluación crítica de la misma, no manifiesta relación 
significativa alguna con las preferencias por métodos instruccionales. Estos resultados apuntan que los sujetos caracterizados por un procesamiento profundo se comportan de este modo independientemente del formato instruccional utilizado por el profesor y sin mostrar preferencias significativas hacia una u otra metodología instruccional. Del mismo modo, tampoco hemos encontrado relaciones significativas entre preferencias por métodos instruccionales y la dimensión Estudio Metódico. De este modo, los sujetos caracterizados por el uso sistemático de las técnicas de estudio tradicionales parecen actuar de este modo de forma consistente, independientemente del método instruccional utilizado en las clases y de sus preferencias por unos u otros métodos instruccionales. Estos resultados también coindicen básicamente con los referenciados en la investigación sobre esta dimensión, ya que los sujetos con elevadas puntuaciones en la misma suelen mostrarse como estudiantes cumplidores, trabajadores, moderados y tranquilos (Schmeck y Ribich, 1978).

La dimensión de estilos de aprendizaje Retención de Hechos, que contiene items que indican la preferencia por los contenidos tipo hecho y por el recuerdo de detalles, sí manifiesta una correlación significativa inversa con las preferencias por los métodos multidireccionales -centrados en el aprendiz y con elevados niveles de interacción entre iguales-, no así con los unidireccionales y autónomos. Este resultado también resulta congruente, dado que los métodos multidireccionales se alejan de las perspectivas instruccionales que priman el recuerdo de la literalidad de la información como resultado del proceso de enseñanza/aprendizaje, así como de la utilización de técnicas de evaluación dirigidas a evaluar reconocimiento, recuerdo y/o memorización de definiciones. Por último, la dimensión Procesamiento elaborativo -caracterizada por el uso de imágenes visuales, por la síntesis de la información presentada en clase, por la búsqueda de relaciones y aplicaciones prácticas de la misma- se relaciona con las preferencias por los métodos unidireccionales y multidireccionales, pero no así con los autónomos. Así, el individuo que puntúa alto en esta dimensión muestra preferencias por ambos tipos de métodos instruccionales, cuestión que puede estar relacionada con una mayor familiarización con los mismos frente a los métodos autónomos, encontrando mayor facilidad para elaborar el material de clase de forma personal, trasladar la información a su propia terminología y/o generar ejemplos concretos de su propia experiencia.

En cuanto a la relación entre las dimensiones motivacionales hacia el aprendizaje y las preferencias por métodos instruccionales de los aprendices, los resultados también muestran 
niveles de asociación significativos aunque de escasa magnitud -en ningún caso superiores a .23- entre ambos constructos.

De forma más específica, la orientación intrínseca hacia las metas/objetivos de aprendizaje se relaciona con las preferencias por métodos unidireccionales y multidireccionales, aunque no así con los autónomos. Dado que una orientación intrínseca indica que los sujetos perciben la realización de las tareas académicas como un fin en sí mismas por razones relacionadas por la curiosidad, competencia o cambio (Pintrich et al., 1991) resulta lógico que muestren preferencias simultáneamente por distintos formatos de metodología instruccional, a excepción de los métodos autónomos en que, al verse implicada habitualmente la utilización de nuevos recursos tecnológicos exigen la familiarización y dominio previo de los mismos para poder centrarse en la adquisición de los conocimientos implicados en las tareas académicas. Sin embargo, una orientación extrínseca se relaciona de forma significativa exclusivamente con las preferencias por los métodos unidireccionales, resultado también lógico dado que los sujetos caracterizados por esta orientación no consideran las tareas académicas como un fin en sí mismas, sino que la razón principal para su realización se refiere a la obtención de buenos resultados, a la competición con los compañeros y/o a la obtención de altos niveles de ejecución. La consecución de estos objetivos parece resultar más fácil en materias que utilizan métodos instruccionales unidireccionales tradicionales que en materias que priman la utilización de métodos basados en la interacción entre pares y/o la utilización-dominio previo de recursos tecnológicos diversos.

Adicionalmente, dos dimensiones motivacionales hacia el aprendizaje (ansiedad ante las evaluaciones y valor de la tarea) no presentan relación significativa alguna con las preferencias por métodos instruccionales. Así, el nivel de ansiedad (pensamientos negativos y activación afectiva y psicofisiológica) y estrés académico ante las evaluaciones, así como grado de importancia y utilidad que los sujetos le conceden a las tareas académicas, parecen manifestarse como una característica individual independiente de su tendencia a elegir o expresar preferencias por una técnica o combinación de técnicas instruccionales determinadas.

Respecto a las creencias sobre el control del aprendizaje (interno frente a externo), las preferencias por métodos unidireccionales y autónomos muestran una relación significativa con una atribución interna de los resultados académicos, cuestión que prácticamente también se produce respecto a los métodos multidireccionales. Así, aunque con niveles de asociación 
de muy escasa magnitud (la correlación superior es de .17), los resultados indican que las preferencias por los distintos métodos instruccionales se relacionan con una percepción de control interna del aprendizaje y con las creencias de que sus esfuerzos se traducirán en resultados académicos positivos. Por último, la percepción de autoeficacia hacia el aprendizaje sólo manifiesta una relación significativa con las preferencias por los métodos unidireccionales. Los resultados también pueden ser considerados congruentes, dado que los sujetos están más familiarizados con esta metodología instruccional, han desarrollado estrategias de actuación más ajustadas y refinadas frente a las misma, está más directamente vinculada a la actuación y esfuerzo individual personal -el nivel de interacción con los pares es menor- y no supone la utilización de recursos y dispositivos tecnológicos -como sucede habitualmente con los métodos autónomos-. En consecuencia, resulta congruente que sus expectativas de éxito y la confianza en sus propias habilidades para cumplimentar las tareas académicas sean superiores con los métodos unidireccionales frente a los multidireccionales y autónomos.

En definitiva, los resultados destacan un nivel de asociación significativo -aunque de reducida magnitud- entre las preferencias de los aprendices por los distintos métodos instruccionales y sus estilos y estrategias motivacionales hacia el aprendizaje. Esta cuestión enfatiza la necesidad de considerar en la planificación de los procesos de enseñanza/aprendizaje y en la selección de la metodología instruccional a emplear en la enseñanza universitaria no sólo las variables contextuales tradicionales (p.e., tipo de contenidos, temporalización y/o preferencias del propio docente) sino también las preferencias de los estudiantes, considerando las características de los mismos en la planificación y desarrollo de los procesos formativos (Ford y Chen, 2001). Por otro lado, también debemos ser conscientes de la importancia de diversificar la metodología instruccional que utilizamos en nuestras clases, dado los diversos efectos positivos para los aprendices: En primer lugar, sobre la motivación y el nivel de aprendizaje al utilizar métodos que se ajusten a sus preferencias sobre metodología de enseñanza; en segundo lugar, a convertirse en estudiantes más expertos y eficaces al interactuar con métodos diversos, ajustados o no a las mismas (Check, 1984; Entwistle \& Peterson, 2005; Loo, 2004; Sadler-Smith y Smith, 2004).

Por último, diversificar los métodos instruccionales en la enseñanza universitaria resulta especialmente importante en el caso de la titulación de Magisterio. Facilitar la experimentación directa de las distintas metodologías de enseñanza, junto a una sólida formación teórica y aplicada respecto a las mismas, favorecerán el desarrollo de las habilidades docentes 
para seleccionar y adaptar las metodologías de enseñanza más adecuadas en función de las características del contexto instruccional y de las características individuales de sus futuros alumnos.

\section{Referencias}

Anderson, L. y Burns, R. (1989). Research in classrooms. Oxford: Pergamon Press.

Ausburn, L. J. y Brown, D. (2006). Learning Strategy Patterns and Instructional Preferences of Career and Technical Education Students. Journal of Industrial Teacher Education, 43(4), 6-39.

Cano, F. y Justicia, F. (1993). Factores académicos, estrategias y estilos de aprendizaje. Revista de Psicología General y Aplicada, 46, 1-12.

Check, J. F. (1984). Teaching learning preferences of the adult learner. Education, 105, 107112.

Clariana, M. (2001). Las preferencias instruccionales: conceptualización y evaluación. Revista de Psicología General y Aplicada, 54 (2), 259-277.

Corno, L. y Snow, R.E. (1986). Adapting teaching to individual differences among learners. En M.C. Wittrock (ed.), Handbook of research on teaching. New York: McMillan, 605-629.

Craik, F. y Lochart, R. S. (1972). Levels of processing . A framework for memory research. Journal of verbal Learning and Verbal Behavior, 11, 671-684.

De la Fuente, J. y Justicia, F. (2007). El modelo DIDEPRO de regulación de la enseñanza y del aprendizaje: Avances recientes. Electronic Journal of Research in Educational Psychology, 13, 5(3), 535-564.

Entwistle, N. (1988). Styles of Learning and Teaching. Londres: David Fulton.

Entwistle, N. y Peterson, E.R. (2005). Conceptions of learning and knowledge in higher education: Relationships with study behaviour and influences of learning environments, International Journal of Educational Research, 41, 407-428.

Fraser, B. (1987). Identifying the salient facets of a model of student learning: A synthesis of metaanalyses. International Journal of Educational Research, 11(2), 187-212.

García-Ros, R.; Pérez-González, F.; Martínez, T. y Alfonso, V. (1999). Validación de una adaptación española del Inventory of Learning Processes: Un análisis con estudiantes de enseñanza secundaria. Revista Portuguesa de Educaçao, 12(2), 261-272. 
Gardner, B.S. y Korth, S. J. (1990). A framework for learning to work in teams. Journal of Education for Business, 74, 28-33.

Gusfstafson, K.L. y Branch, R.M. (2002). What is instructional design? En R.A. Reiser y J.V. Dempsey (eds.), Trends and issues in Instructional Design and Technology, 16-25. New Jersey: Prentice Hall.

Hernández, P. (1998). Diseñar y enseñar. Teoría y técnicas de la programación y del proyecto docente. Madrid: Narcea.

Hocevar, D.; Zimmer, J. y Strom, B. (1987). The measurement of preference for course structure and preference for course difficulty: The Instructional Preferences Inventory (IPI). Educational and Psychological Measurement, 47(4), 997-1003.

Honey, P. \& Mumford, A. (1992). The manual of Learning Styles. Maidenhead: Peter Honey.

James, N. (1962). Personal preference for method as a factor in learning. Journal of Educational Psychology, 53(1), 43-47.

Kolb, D. A. (1984). Experience as the source of learning and development. Englewood Cliffs: Prentice-Hall.

Kolb, D. A. (1985). The learning style inventory: Technical manual.Boston: McBer.

Loo, R. (2004). Kolb's Learning Styles and Learning Preferences: Is There a Linkage? Educational Psychology, 24(1), 99-108.

Lowyck, J.; Elen, J. y Clarebout, G. (2005). Instructional conceptions: A prospective analysis. International Journal of Educational Research, 41, 429-444.

Marqués, P. (2000) (on line). Investigación sobre TIC en educación.

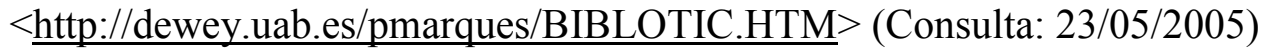

Monereo, C. (2007). Hacia un nuevo paradigma del aprendizaje estratégico: El papel de la mediación social, del self y de las emociones. Electronic Journal of Research in Educational Psychology, 13, 5(3), 497-534.

Newby, T.J.; Stepich, D.A.; Lehman, J.D. y Russell, J.D. (2000). Instructional Technology for Teaching and Learning. New Jersey: Prentice-Hall.

Phillips, F. (1999). Business Students' Learning Preferences and Associated Task Performance. Journal of Education for Business, 75(1), 27-32.

Pintrich, P.; Smith, D. A.; Garcia, T. y McKeachie, W. J. (1991). A manual for the use of the Motivated Strategies for Learning Questionnaire. Michigan: University of Michigan.

Podolskij, A.I. (1997). Instructional design for schooling. En S. Dijkstra, N. Seel, F. Schott y R.D. Tennyson (eds.), Instructional design: International perspective, vol 2, 289-314. New Jersey: Lawrence Erlbaum. 
Reigeluth, C.M. (1999). What is instructional-design theory and how is it changing? En C.M. Reigeluth (ed.), Instructional design. Theories and models, vol. II, 5-29. New Jersey: Lawrence Erlbaum.

Reigheluth, C.M. y Moore, J. (1999). Cognitive education and the cognitive domain. En C.M. Reigeluth (ed.), Instructional design. Theories and models, vol. II, 51-68. New Jersey: Lawrence Erlbaum.

Reynaldo, J. y Galán, F. (2000). Estrategias de aprendizaje, motivación y rendimiento en alumnos universitarios. Revista de orientación y psicopedagogía, 11, 35-50.

Rivas, F. (1997). El proceso de Enseñanza/Aprendizaje en la situación educativa. Barcelona: Ariel.

Roces, C.; Tourón, J.; González, M.; Núñez, J.; González, J. y García, M. (1993). Adaptación de la escala motivacional del MSLQ. Actas congreso Iberoamericano Psicología, 1-3.

Romiszowski, A.J. (1981). Designing Instructional Systems: Decision-making in course planning and curriculum design. Londres: Kogan Page.

Sadler-Smith, E. (1996). Learning styles: a holistic approach. Journal of European Industrial Training, 20, 29-36.

Sadler-Smith, E. (1997). "Learning style": Frameworks and instruments. Educational Psychology, 17, 51-63.

Sadler-Smith, E. y Riding, R. (1999). Cognitive style and instructional preferences. Instructional Science, 27, 355-371.

Sadler-Smith, E. y Smith, P.J. (2004). Strategies for accommodating individuals' styles and preferences in flexible learning programmes. British Journal of Educational Technology, 35(4), 395-412.

Schmeck, R. R. (1983). Learning styles of college students. In R. Dillon \& R. Schmeck (eds.): Individual differences in cognition. N. Y.: Academis Press.

Schmeck, R. R. (1988). Individual differences and learning strategies. In C. E. Weinstein, E. T. Goetz \& P. A. Alexander (eds): Learning and study strategies: Issues in Assessment, and Evaluation. San Diego. Academic Press.

Schmeck, R. R. y Ribich, F. (1978). Construct validation of the Inventory of Learning Processes. Applied Psychological Measurement, 2, 551-562.

Schmeck, R. R.; Ribich, F. y Raamanaiah, N. (1977). Development of a self-report inventory for assessing individual differences in learning processes. Applied Psychological Measurement, 1, 413-431. 
Shuell, T. J. y Farber, S.L. (2001). Students' perceptions of technology use in college courses. Educational Computing Research, 24(2), 119-138.

Smith, P. J. (2001). Technology student learning preferences and the design of flexible learning programs. Instructional Science, 29, 237-254.

Tait, H.; Entwistle, N.J. y McCune, V. (1998). ASSIST: A re-conceptualisation of the approaches to studying inventory. In C. Rust (Ed.), Improving student learning: Improving students as learners, 262-271. Oxford Brookes University: Oxford.

Terlouw, A.I. (1997). Instructional design in Higher Education. En S. Dijkstra, N. Seel, F. Schott y R.D. Tennyson (eds.), Instructional design: International perspective, vol 2, 341-368. New Jersey: Lawrence Erlbaum.

Van den Bosch, H. (2006). Personalized Course Delivery: An Empirical Analysis of the Feasibility of Adapting Distance Learning Courses to Individual Students' Learning Preferences. Industry and Higher Education, 20(5), 337-345. 